\section{Mild hypertension: MRC working party report}

The results of further analysis of the Medical Research Council's trial of drug treatment of mild hypertension (4 June, p 1565) contain a major statement that is not reflected in the abstract or in the discussion: that mild hypertension with a sustained diastolic pressure of $90-109 \mathrm{~mm} \mathrm{Hg}$ was not a significant risk factor for stroke or myocardia infarction for women aged 35-64. The abstract, however, implies that treatment of women will prevent stroke, and these statements are incompatible.

There is also an inconsistency in the analysis of the number of strokes prevented in high risk male smokers aged 55-64 with high systolic pressure at entry. In the discussion four high risk patients are said to need treatment for five years to avoid one stroke. It is then stated that 100 men in the highest risk group observed without treatment for five years would be expected to suffer five strokes. This is a fivefold difference even assuming that treatment was completely successful in preventing stroke.

It would be interesting to know how body mass index changed from being a significant risk facto in 1985 to making no significant contribution in 1988.

I welcome the Medical Research Council's effort to produce clear guidelines but think that further clarification is needed.

JOHN KNOX

Department of Medicine for the Elderly,

North Humberside,

Kingston General Hospital,

Hull HU3 IUR

The Medical Research Council's working party on the treatment of mild hypertension has estimated the rates for cardiovascular events in low and high risk patients with blood pressures of $160 / 110 \mathrm{~mm}$ $\mathrm{Hg}$ and also the benefits that can be achieved by antihypertensive treatment. It is, however, doubtful whether it is legitimate to generalise so confidently from the results of the MRC trial.

Nowhere does the report mention that the patients treated with placebo in this study had a mean initial diastolic pressure during the study of only $91-92 \mathrm{~mm} \mathrm{Hg}$-that is, that many of the patients were not really hypertensive. This pressure, which was considerably lower than that found at the screening examinations at entry, seems to have been found as soon as the subjects pressures were measured by their own doctors. Which measurement, the exaggeratedly high pressure on screening at entry or that recorded by the general practitioners, does the diastolic pressure of $110 \mathrm{~mm} \mathrm{Hg}$ represent?

Similarly, nowhere does the report mention that $18 \%$ of the men and $13 \%$ of the women allocated to the placebo group were treated for ethical reasons because their diastolic pressures rose to $110 \mathrm{mg} \mathrm{Hg}$ or more. ${ }^{1}$ According to Miall and Greenberg, the mortality in these subjects was less than that in the untreated controls matched for age and sex whose pressures remained within the trial range.' They comment: "By continuing to analyse them as controls we are diluting the trial and tending to mask differences between treated [subjects] and controls. We are thus introducing a bias against a positive result." Does the working party really no think that this is germane to the question of estimated risk and benefit? Further, how can the risk caused by a diastolic pressure of $110 \mathrm{~mm} \mathrm{Hg}$ be gauged when people who developed this pressure were given treatment?

Finally, nowhere does the report mention that the subjects were volunteers (who are often healthier than the populace in general) and largely

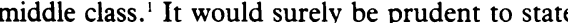
that the estimates of risks and benefits derived from the trial data can be applied safely only to this type of patient.

The prestige of the Medical Research Council's trial is such that its findings are given great weight. I hope that the working party will recalculate its data, taking the first two points into consideration, and will emphasise the third point in further reports. There is a danger that hypertension will get an undeservedly benign reputation.

Wellcome Medical Research Institute,

F O SIMPSON

Dunedin,

New Zealand

1 Miall WE, Greenberg G. Mild hypertension-is there pressure to treat? ' Cambridge: University Press, 1987.

AUTHORS' REPLY, - We are glad of the opportunity to comment on Dr Knox's points about the relation between blood pressure and the risk of cardiovascular disease and about the effects of treatment in women. Epidemiological studies and insurance data leave no doubt about the increase in risk as blood pressure rises. Our paper confirmed this relation in men. In the women studied the relative risk of myocardial infarction for a $10 \mathrm{~mm} \mathrm{Hg}$ increase in systolic blood pressure was $1 \cdot 15$, and for stroke a $4 \mathrm{~mm} \mathrm{Hg}$ increase in diastolic pressure conferred a relative risk of $1 \cdot 22$. That these relations were not significant at a conventional level almost certainly reflects the rather small numbers of myocardial infarctions and strokes among the women. We drew attention to the possibility that the beneficial effect of bendrofluazide on the rate of stroke extends beyond the benefit that can be attributed to its hypotensive effect. For this reason also, our findings on the relation of blood pressure, risk, and the effects of treatment are compatible. In quantifying the strokes prevented we illustrated the benefits of treatment in two ways. Firstly, we took a hypothetical example of a man aged 65 with rather extreme risk factor values to show what might be expected in such patients. Elsewhere we used the observed results in a group of patients at high risk who were, however, not only somewhat younger but also not exposed to the extreme risk in our first example. The estimate based on the experience of the group is inevitably approximate, bearing in mind the small numbers of events available for analysis, but we expect it to differ noticeably from that derived from the high risk person. The explanation of Dr Knox's final point is that patients were categorised as obese or non-obese in many of the analyses in our first report,' though (as we pointed out) body mass index was included as a continuous variable in the analyses in our recent paper. This difference in procedure accounts for the apparent difference in the significance of body mass index as a risk factor.

Dr Simpson has misrepresented our account of the course of blood pressure during the trial. Our first report clearly showed the fall in blood pressure over the first few months in both treated and placebo groups.' The increased risk associated with initial blood pressures of $160 / 110 \mathrm{~mm} \mathrm{Hg}$, which we used in some of our examples, is of course well established in many other studies, even though the effects of habituation and regression to the mean result in lower readings subsequently. Further, the trial was instituted to assess potential benefits in mild hypertension, otherwise known as labile hypertension or, preferably, labile blood pressure, as this presented an important clinical problem and probably still does. Against this background in clinical practice decisions about drug treatment are usually made only after several measurements of blood pressure. To establish risk and in conducting a large scale trial (as well as for many patients in clinical practice) it would be unwise to regard patients who had sustained increases in diastolic pressure during screening and entry procedures as "not really hypertensive." Throughout, we have made it clear that our results are based on analysis of "intention to treat." Though these analyses tend to reduce real treatment effects, we pointed out in our first report that there were no clear differences between the results of the analyses of "intention to treat" and of "treatment." Finally, we reject Dr Simpson's suggestion that the population of the trial has been inadequately described. We have never disguised the tendency towards the overrepresentation of social classes I, II, and III by comparison with the country as a whole. Participation in the trial was voluntary and the result of invitations to screening examinations to all patients in the relevant age groups in the practices concerned and not, as Dr Simpson seems to imply, the result of a call for "volunteers." Inevitably, participants in any trial are to some extent selected, particularly when clinical reasons for exclusion are also taken into account. Though the magnitude of the benefits of treatment might to some extent differ among groups not fully represented in our trial, it seems unlikely that qualitatively different effects would have been established.

STANLEY PEART T W MEADE

MRC Epidemiology and Medical Care Unit, Northwick Park Hospital,

Harrow,

Middlesex HA1 3UJ

1 Medical Research Council Working Party. MRC trial of treat ment of mild hypertension: principal results. $\mathrm{Br} \mathrm{Med} \mathcal{J}$ $1985 ; 291: 97-104$

\section{General practitioner referral rates}

In their discussions on the referral rates of general practitioners Dr Martin Roland (13 August, p 437) and Dr Marshall Marinker and others (13 August, p 461) rightly point out the complexity of defining a high referrer. The table comparing the workload of a trainer and his trainee illustrates this point

Numbers of consultations and referrals by trainee and trainer from 23 August 1987 to 31 fanuary 1988

\begin{tabular}{lrr}
\hline & Trainee & Trainer \\
\hline Consultations & 1257 & 1881 \\
Total referrals & 59 & 99 \\
Referrals for ear, nose, and throat & 17 & 2 \\
$\begin{array}{l}\text { Consultations related to ear, nose, } \\
\quad \text { and throat }\end{array}$ & 365 & 166
\end{tabular}

When referrals as a whole are considered the trainer referred $67 \%$ more patients but if an allowance is made for the higher rate of consultation the excess was only $12 \%$, which is not significant ( $\chi^{2}$ with Yates's correction). The trainee, however, referred far more cases to the local ear, nose, and throat department, and this excess was significant when compared with the rates of consultation ( $p>0 \cdot 001)$. Further analysis shows a substantial excess of consultations by the trainee in this subject. If the referrals are now compared with the rates of consultation in this subject the ratio drops to 3.88 , but more importantly the difference becomes nonsignificant $(\mathrm{p}>0.05)$.

Notwithstanding the limited scope of these data this analysis shows that a marked difference in referrals between two doctors working in the same practice becomes insignificant when rates of consultation by subject are taken into account and re-emphasises the fact that confidence intervals are wide. Review of each of the 19 patients referred to the ear, nose, and throat department showed that each referral was appropriate. As the trainee had held a post in ear, nose, and throat surgery locally immediately before his traineeship the hypothesis 
that greater experience results in more frequent referral is supported.

Before any attempt can be made to improve the cost-benefit equation for referral further study needs to be made into doctors' patterns of workload and experience and their effect on rates of referral.

If a fair assessment is to be made of an individual doctor's rates of referral the work entailed may outweigh the benefits. Moreover, such close scrutiny of a doctor's work is bound to be perceived as threatening by some, all the more so if it is imposed rather than performed voluntarily.

C R PEARSON

Suffolk NR35 ILQ

\section{Mortality in women in relation to childbearing}

Dr Adèle Green and colleagues (6 August, p 391) claim that because standardised mortality ratios for diabetes increase with parity this is evidence for an association of the incidence of diabetes with multiparity. Leaving aside the small number of deaths ascribed to diabetes (What happened to the editorial enthusiasm for confidence intervals?) and the fallibility of death certificates in relation to diabetes, ${ }^{1}$ the discussion of relevant published studies is wanting. The subject was extensively reviewed by West in $1978^{2}$ and seems to have disappeared since. West found the results to be conflicting, with most reports failing to establish an association between parity and diabetes or glucose tolerance. Dr Green and colleagues quote in support of their thesis the view "that pregnancy exaggerates the metabolic defect of diabetes and may cause it to appear first as gestational diabetes." Harris, has, however, recently presented strong evidence against the view that gestational diabetes has anything other than a temporal relation with pregnancy. Antenatal case finding simply increases the chance that diabetes will be recognised, and multiple pregnancies further increase the probability. Thus the conclusions with regard to diabetes rest on slender foundations.

R J JARRETT

Department of Community Medicine,

United Medical and Dental Schools of

Guy's and St Thomas's Hospitals,

Guy's Hospital,

London SE1 9RT

1 Fuller JH, Elford J, Goldblatt P, Adelstein AM. Diabetes mortality: new light on an underestimated public health problem. Diabetologia 1983;24:336-41.

2 West KM. Epidemiology of diabetes and its vascular lesions. New York: Elsevier, 1978:221-4.

3 Harris MI. Gestational diabetes may represent discovery of preexisting glucose intolerance. Diabetes Care 1988;11:402-11.

\section{Ultrasonography for diagnosing appendicitis}

Dr R H Pearson concludes that ultrasonography for diagnosing appendicitis should not be used routinely but only in patients in whom the clinical diagnosis is equivocal (30 July, p 309). This conclusion suggests that it is possible on clinical grounds to separate those patients who possibly have appendicitis from those who certainly have it. This selection, however, is illusory as the clinical diagnosis is never certain.

In a recent article in this journal a similar selection was attempted.' In the group whose need for an appendicectomy was considered clinically evident the negative appendicectomy rate was still $22 \%$.

In our hospital we have practised ultrasonography routinely in all patients with suspected appendicitis for three years. We confirm that ultrasonography is most useful in those patients whose clinical symptoms are atypical, yet we continue to perform ultrasonography routinely in all patients, and also in those whose need for an appendicectomy seems clinically pertinent. We do so because in $10 \%$ of those whose need for an appendicectomy is clinically obvious ultrasonography still leads to an essential change in therapeutic management by reliably identifying another condition as the cause of the patient's symptoms - for example, Crohn's disease, gynaecological conditions, bacterial enteritis caused by campylobacter or yersinia, perforated peptic ulcers, or cholecystitis. ${ }^{2.4}$

Another important advantage of liberally using ultrasonography is that it enables the radiologist to acquire the necessary skill to offer a reliable diagnosis.

In our prospective study of 111 consecutive patients with suspected appendicitis only four had a normal appendix removed and only one experienced a delay in hospital. ${ }^{5}$ If performed by an experienced radiologist ultrasonography can be immensely beneficial and should not be withheld from any patient with clinical signs of appendicitis.

J B C M PUYLAERT

Department of Radiology,

Westeinde Hospital

Lijnbaan 32,

The Hague,

The Netherlands

P.terson-Brown S, Thompson JN, Eckersley JRT, Ponting GA Dudley HAF. Which patients with suspected appendicitis Dudley HAF. Which patients with suspected append
should undergo laparoscopy? $\mathrm{Br}$ Med $\mathcal{F}$ 1988;296:1363-4.

Puylaert JBCM. Mesenteric adenitis and acute terminal ileitis: US evaluation using graded compression. Radiology 1986;161: 691-5.

3 Puylaert JBCM, Lalisang RI, Van der Werf SDJ, Doornbos L. Campylobacter ileocolitis mimicking acute appendicitis: differentiation by graded-compression US. Radiology 1988 166:737-40.

Puylaert JBCM. The use of ultrasound in patients with clinical signs of appendicitis [Doctoral thesis]. Leiden: University of Leiden, 1988. $91 \mathrm{pp}$

5 Puylaert JBCM, Rutgers PH, Lalisang RI, et al. A prospective study of ultrasonography in the diagnosis of appendicitis. N Engl f Med 1987;317:666-9.

\section{Consensus on HIV testing}

We have followed the debate about the need to obtain patients' permission to test for the human immunodeficiency virus (HIV) (13 August, p 486). As consultants working in a regional neurological unit we are faced with considerable problems. AIDS may present with dementia, meningitis, encephalopathy, cerebrovascular disease, disease of the spinal cord, neuropathy-for example, Guillain-Barré syndrome-and polymyositis, to mention a few; if we have to obtain permission for testing in most neurological cases this will be time consuming. Recent data also suggest that AIDS can affect the central nervous system in fairly minor ways, for instance presenting as Bell's palsy. We have no doubt that most neurologists and other busy practitioners will not discuss the possibility of AIDS in patients with such symptoms. We also do not wish to distress most of our patients, who will require testing but will be found to be negative. We are sure that we are not alone in this. The results will be that patients positive for HIV will not be identified and will continue to spread the disease, leading to a considerable underestimate of its prevalence. We are also surprised by proposals to screen pregnant women for HIV to get a better idea of the spread of the disease; this is probably the group of people least likely to have the disease, except for children under 10 and pensioners.

We see no difference in testing for AIDS and for other serious and less serious conditions-for instance, electroencephalography of rare patients whose "dizzy spells" are due to epilepsy. Are we to ask such patients whether we can do the test? The diagnosis may lose them their livelihoods and their cars, and their insurance may be loaded. Are we to ask for permission and then not do the test if patients, not unreasonably from their viewpoint refuse?

When a patient with symptoms asks a doctor for help is it not implicitly understood that the doctor will perform appropriate investigations to identify a cause, even if such a cause has adverse effects on the patient's future prospects? Though we would obtain specific written consent for all diagnostic procedures associated with appreciable risk, do we now need consent for harmless tests whose results may have serious implications?

Are we to accept that neurological illnesses associated with HIV will be diagnosed only if the patient is already known to be positive for HIV from some previous non-neurological illness or if the neurological illness is so suggestive of HIV that to miss it would be negligent? Would it even be construed as negligent if all such patients presenting with neurological symptoms were not tested for HIV?

What a sad state of affairs this is, and one particularly worrying for patients, relatives, and health service staff, who may be placed needlessly at risk. It will be even more tragic if and when useful therapeutic agents become available.

$\begin{array}{rr}\text { A BOWDEN } & \text { IAN R WILLIAMS } \\ \text { L D BLUMHARDT } & \text { G FINDLAY } \\ \text { DAVID CHADWICK } & \text { PATRICK FOY } \\ \text { P R D HUMPHREY } & \text { RICHARD JEFFREYS } \\ \text { B R F LECKY } & \text { J B MILES } \\ & \text { M SHAW }\end{array}$

Mersey Regional Department of Medical and

Surgical Neurology,

Walton Hospital,

Liverpool L9 $1 \mathrm{AE}$

\section{Cervical intraepithelial neoplasia}

Dr Robinson and her colleagues call for the introduction of cytological screening for sexually active girls under the age of 16 ( 30 July, p 359). Surely we have enough difficulties with the cervical screening programme without extending it solely on the basis of observations on a single cohort. If the authors wish to start cervical screening in girls under 16 because such screening "is useful to educate them" then the costs, risks, and benefits of this educational technique need careful investigation, particularly in view of the increasing evidence about the risks of cervical screening and referral for colposcopy. ${ }^{1}$ If screening is to be introduced to reduce the risk of cervical intraepithelial neoplasia then the screening test should, like all screening tests, be formally evaluated by randomised controlled trials, so that it can be compared with the criteria set out in the classic papers on screening published in the $1960 \mathrm{~s}$, which are equally relevant today.

Community Health Offices,

J A M GRAY

\section{Radcliffe Infirmary,}

Oxford OX26HE

1 Jordan JA. Minor degrees of cervical intraepithelial neoplasia. BrMed J 1988;297:6.

\section{The monstrous regiment}

Jane Salvage writes of the discrimination against women doctors found by the Policy Studies Institute and comments on similar problems in nursing (13 August, p 494). Although I believe such discrimination exists, the behaviour of many women is also a contributory factor.

My partnership recently advertised for a full time partner. We received applications from four women and 94 men, despite the preponderance of women choosing vocational training. Women doctors are in the fortunate position of commanding an income which allows them to obtain the best 